\title{
$h$-STABILITY AND BOUNDEDNESS RESULTS FOR SOLUTIONS TO CERTAIN NONLINEAR PERTURBED SYSTEMS
}

\author{
HANEN DAMAK, MOHAMED ALI HAMMAMI AND ABIR KICHA
}

\begin{abstract}
In this work, we establish some new sufficient conditions to show the uniform $h$-stability for nonlinear time-varying perturbed systems which can be viewed as an extension of the uniform exponential stability and polynomial stability. Also, we study the boundedness of solutions when the origin is not necessarily an equilibrium point of the perturbed system. The idea is to use some Gronwall type integral inequalities. As an illustration, we present some examples with simulations to show the applicability of the obtained results.
\end{abstract}

\section{INTRODUCTION}

In the study of stability properties of differential and difference systems, a new concept of stability was introduced by Pinto in $[14,15]$ called $h$-stability, which is stronger than exponential stability. The intention of this notion is to obtain stability results for a weakly system under some perturbations when the origin is an equilibrium point. Medina and Pinto in [13] extended the study of exponential asymptotic stability to a variety of reasonable systems called $h$-systems. When we investigate the asymptotic stability, it is not easy to work with non-exponential types of stability. Because of that, the concept of $h$-stability is very useful and more general in the study of differential systems. This theory has been developed very intensively and several works are published, see [4-6]. However, when the origin is not necessarily an equilibrium point, the desired system may be unstable and yet the system may oscillate sufficiently near this state so that its performance is acceptable and still possible to analyze the asymptotic of solutions with respect to a small neighborhood of the origin, which yields to the concept of practical stability, see $[1,2,10]$.

In this paper, we investigate the concept of uniform boundedness of certain time-varying perturbed systems. This notion has been studied by using Lyapunov analysis in $[6,12]$ and here we use a technique called Gronwall's inequalities, see [7]. In mathematics, this kind of inequalities allows one to bound a function that is known to satisfy a certain differential or integral inequality by the solution to the corresponding differential or integral equation. This type of inequalities can be used in the study of existence, uniqueness and stability properties of solutions

$M S C$ (2010): primary 37C75, 37B25.

Keywords: Gronwall's inequalities, perturbed systems, uniform $h$-stability, uniform boundedness. 
to differential perturbed equations (see $[8,9]$ ). This work focuses on the study of the global uniform $h$-stability and the global uniform boundedness of time-varying perturbed differential equations under conditions on the perturbed terms and we will see how we can use the Gronwall's inequalities in this research.

The remainder of this paper is organized as follows. In Section 2, some preliminary results are summarized. The main aim of this paper is provided in Section 3 and 4 . Some examples are carried out in Section 5 to show the applicability of the proposed method. Finally, our conclusion is given in Section 6 .

\section{MATHEMATICAL PRELIMINARIES}

In the present section, we introduce some basic definitions and preliminary facts which we need in the sequel. First, we adopt the following notations:

- $\mathbb{R}_{+}=[0,+\infty)$.

- $\mathbb{R}^{n}$ the real $n$-dimensional Euclidean space and $\|\cdot\|$ stands for its Euclidean vector norm.

- $\mathbb{R}^{n \times n}$ the set of all $n \times n$ matrices whose entries are real valued.

- For $r \geq 0, \mathcal{B}_{r}$ denotes the closed ball of $\mathbb{R}^{n}$ centred at zero, i.e., $\mathcal{B}_{r}:=\left\{x \in \mathbb{R}^{n}\right.$ : $\|x\| \leq r\}$.

We consider now the nonlinear time-varying differential system:

$$
\dot{x}=f(t, x), \quad x\left(t_{0}\right)=x_{0}, \quad t \geq t_{0} \geq 0,
$$

where $x \in \mathbb{R}^{n}$ is the state and $f: \mathbb{R}_{+} \times \mathbb{R}^{n} \rightarrow \mathbb{R}^{n}$ is continuous in time and locally Lipschitz in $x$, uniformly in $t$.

Let $x(t)=x\left(t, t_{0}, x_{0}\right)$ be denoted by the unique solution to system (2.1) passing through the initial state $x_{0} \in \mathbb{R}^{n}$ at time $t=t_{0}$.

The following definitions state the known different types of stability taken from $[12]$.

Definition 2.1. The system (2.1) is said to be uniformly stable if for each $\varepsilon>0$ there is $\delta=\delta(\varepsilon)>0$, such that

$$
\left\|x\left(t_{0}\right)\right\|<\delta \Longrightarrow\|x(t)\|<\varepsilon, \quad \forall t \geq t_{0} \geq 0 .
$$

Definition 2.2. The system (2.1) is said to be globally uniformly exponentially stable, if there exist positive constant numbers $\lambda_{1}$ and $\lambda_{2}$, such that for each $x_{0} \in \mathbb{R}^{n}$ and for any $t_{0} \in \mathbb{R}_{+}$

$$
\|x(t)\| \leq \lambda_{1}\left\|x_{0}\right\| e^{-\lambda_{2}\left(t-t_{0}\right)}, \quad \forall t \geq t_{0} .
$$

The constant $\lambda_{2}$ is called the convergence rate.

Now, we recall the notion of global uniform $h$-stability when the origin is an equilibrium point of system (2.1).

Definition 2.3. (See [15]) The system $(2.1)$ is called globally uniformly $h$ stable if there exist $c \geq 1$ and a positive continuous bounded function $h$ on $\mathbb{R}_{+}$, such that for each $x_{0} \in \mathbb{R}^{n}$, we have

$$
\|x(t)\| \leq c\left\|x_{0}\right\| h(t) h\left(t_{0}\right)^{-1}, \quad \forall t \geq t_{0} \geq 0 .
$$


Here, $h(t)^{-1}=\frac{1}{h(t)}$.

Remark 2.4. The $h$-stability property (2.2) includes concepts of uniform exponential stability. The next example illustrates $h$-stable systems without exponential decay.

Example 2.5. Consider the scalar equation:

$$
\dot{x}=-\frac{1}{1+t} x, \quad x \in \mathbb{R}, \quad t \geq 0 .
$$

The solution to the system is given by:

$$
x(t)=\frac{1+t_{0}}{1+t} x\left(t_{0}\right), \quad \forall t \geq t_{0} .
$$

We can see that the system (2.3) is globally uniformly $h$-stable with

$$
h(t)=\frac{1}{1+t} .
$$

On the contrary, if we suppose that (2.3) is exponentially stable, then there exists $\alpha>0$, such that

$$
\left(1+t_{0}\right) \leq e^{-\alpha\left(t-t_{0}\right)}(1+t), \quad \forall t \geq t_{0} .
$$

For $t_{0}=0$ and $t \longrightarrow+\infty$, we obtain a contradiction and hence the system (2.3) is not exponentially stable.

Figure 1 shows the $h$-stability property for system $(2.3)$ with $x(0)=5$.

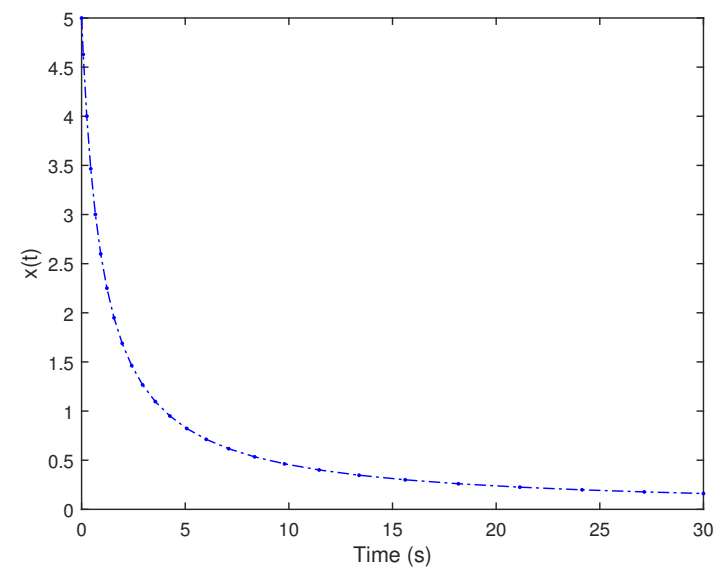

Figure 1. Time evolution of the state $x(t)$ of system (2.3).

Next, we consider the linear time-varying system:

$$
\dot{x}=A(t) x, \quad x\left(t_{0}\right)=x_{0}, \quad t \geq t_{0} \geq 0,
$$

where $A(\cdot) \in \mathbb{R}^{n \times n}$ is a matrix whose elements are continuous functions on $\mathbb{R}_{+}$.

We define the norm of matrices by:

$$
\|A\|_{i}:=\max _{\|x\| \leq 1}\|A x\| .
$$


The stability of the origin of system (2.4) can be characterized by the state transition matrix $R\left(t, t_{0}\right)$ associated with $A(\cdot)$ by the following form:

$$
x(t)=R\left(t, t_{0}\right) x_{0}, \quad x_{0} \in \mathbb{R}^{n}, \quad t \geq t_{0} \geq 0 .
$$

With the aid of this explicit characterization of the solutions to system (2.4), it is possible to derive some useful conditions for the stability of the origin. Since these conditions involve the state transition matrix $R\left(t, t_{0}\right)$, they are not of much computational value, because, in general, it is impossible to derive an analytical expression for $R\left(t, t_{0}\right)$. Nevertheless, they are of conceptual value, enabling one to understand the mechanisms of stability and instability in linear systems.

Next, we define the global uniform asymptotic stability of system (2.4) in terms of $R\left(t, t_{0}\right)$, see [16].

Definition 2.6. The origin is globally stable if and only if, for each $t_{0} \geq 0$, it is true that

$$
\sup _{t \geq t_{0}}\left\|R\left(t, t_{0}\right)\right\|_{i} \leq m\left(t_{0}\right)<+\infty .
$$

Remark 2.7. Note that, in the case of linear systems, the instability does indeed imply that some solution trajectories actually blow up. This is in contrast to the case of nonlinear systems, where instability of the origin can be accompanied by the boundedness of all solutions.

Definition 2.8. The origin is globally uniformly stable if and only if

$$
\sup _{t_{0} \geq 0} \sup _{t \geq t_{0}}\left\|R\left(t, t_{0}\right)\right\|_{i} \leq m_{0}<+\infty .
$$

Definition 2.9. The origin is globally uniformly asymptotically stable if and only if

$$
\begin{gathered}
\sup _{t_{0} \geq 0} \sup _{t \geq t_{0}}\left\|R\left(t, t_{0}\right)\right\|_{i} \leq m_{0}<+\infty \\
\left\|R\left(t+t_{0}, t_{0}\right)\right\|_{i} \rightarrow 0 \quad \text { as } \quad t \rightarrow+\infty, \quad \text { uniformly in } t_{0} .
\end{gathered}
$$

Lemma 2.10. (See [12]) The system (2.4) is globally uniformly asymptotically stable if and only if the state transition matrix satisfies the inequality

$$
\left\|R\left(t, t_{0}\right)\right\|_{i} \leq \lambda_{1} e^{-\lambda_{2}\left(t-t_{0}\right)}, \quad \forall t \geq t_{0} \geq 0,
$$

for some positive constants $\lambda_{1}$ and $\lambda_{2}$.

The next lemma introduces the global uniform $h$-stability in terms of $R\left(t, t_{0}\right)$. It is very effective to show global uniform $h$-stability for linear time-varying systems.

Lemma 2.11. The system (2.4) is globally uniformly $h$-stable if and only if there exist $c \geq 1$ and a positive continuous bounded function $h$ on $\mathbb{R}_{+}$, such that

$$
\left\|R\left(t, t_{0}\right)\right\|_{i} \leq \operatorname{ch}(t) h\left(t_{0}\right)^{-1}, \quad \forall t \geq t_{0} \geq 0 .
$$
have

Proof. Suppose that the system (2.4) is globally uniformly $h$-stable, then we

$$
x(t)=R\left(t, t_{0}\right) x_{0}, \quad x_{0} \in \mathbb{R}^{n}, \quad t \geq t_{0} \geq 0
$$

and the inequality (2.2) holds, then

$$
\left\|R\left(t, t_{0}\right)\right\|_{i}=\max _{\|y\| \leq 1}\left\|R\left(t, t_{0}\right) y\right\|=\max _{\|y\| \leq 1}\left\|x\left(t ; t_{0}, y\right)\right\| \leq \operatorname{ch}(t) h\left(t_{0}\right)^{-1}, \quad \forall t \geq t_{0} \geq 0 .
$$


Hence,

Conversely, we have

$$
\left\|R\left(t, t_{0}\right)\right\|_{i} \leq \operatorname{ch}(t) h\left(t_{0}\right)^{-1}, \quad \forall t \geq t_{0} \geq 0 .
$$

$$
\|x(t)\|=\left\|R\left(t, t_{0}\right) x_{0}\right\| \leq\left\|R\left(t, t_{0}\right)\right\|_{i}\left\|x_{0}\right\| \leq c\left\|x_{0}\right\| h(t) h\left(t_{0}\right)^{-1}, \quad \forall t \geq t_{0} \geq 0 .
$$

Therefore, the system (2.4) is globally uniformly $h$-stable.

Remark 2.12. In linear systems there is the notion of an upper function which is related to upper Lyapunov exponent, see $[3,11,17,18]$.

Definition 2.13. (See [18]) A bounded function $\mu(t)$ is an upper function for system (2.4) if there exists a constant $\lambda$, such that

$$
\|R(t, s)\| \leq \lambda \exp \left(\int_{s}^{t} \mu(\tau) d \tau\right), \quad \forall t \geq s,
$$

where $R(t, s)$ is the state transition matrix of the system.

Remark 2.14. In the case where $\mu$ is an upper function and

$$
h(t) h\left(t_{0}\right)^{-1}=\exp \left(\int_{t_{0}}^{t} \mu(\tau) d \tau\right),
$$

the considered system is $h$-stable and vice versa.

If the origin is not an equilibrium point of the system, we cannot study the problem as a question of stability of equilibrium, which brings us to the concept of uniform boundedness.

Definition 2.15. (See [12]) A solution to (2.1) is said to be globally uniformly bounded if for every $a>0$ there exists $c=c(a)$, such that for all $t_{0} \geq 0$

$$
\left\|x_{0}\right\| \leq a \Longrightarrow\|x(t)\| \leq c, \quad \forall t \geq t_{0} .
$$

Now, we introduce the following technical lemmas that will be crucial in establishing the main results of this work.

Lemma 2.16. (Gronwall-Bellman type integral inequality, see [9]) Let $\varphi, \lambda$ and $\varrho$ be non-negative continuous functions on $\mathbb{R}_{+}$for which the following inequality holds.

$$
\varphi(t) \leq d+\int_{t_{0}}^{t}[\lambda(s) \varphi(s)+\varrho(s)] d s, \quad d \geq 0, \quad \forall t \geq t_{0} .
$$

Then,

$\varphi(t) \leq d \exp \left(\int_{t_{0}}^{t} \lambda(s) d s\right)+\kappa \exp \left(\int_{t_{0}}^{t}\left[\lambda(s)+\frac{1}{\kappa} \varrho(s)\right] d s\right), \quad \forall t \geq t_{0}, \quad \forall \kappa>0$.

Lemma 2.17. (Gronwall inequality) Let $\lambda$ and $\varphi$ be non-negative continuous functions on $\mathbb{R}_{+}$, such that

$$
\varphi(t) \leq d+b \int_{t_{0}}^{t} \lambda(s) \varphi(s) d s, \quad \forall t \geq t_{0},
$$

where $b$ and $d$ are positive constants. Then,

$$
\varphi(t) \leq d \exp \left(b \int_{t_{0}}^{t} \lambda(s) d s\right), \quad \forall t \geq t_{0}
$$




\section{3. $h$-STABILITY ANALYSIS}

In this section, we focus on the study of $h$-stability of solutions to perturbed systems described by the following form:

$$
\dot{x}=A(t) x+B(t) x+F(t, x), \quad t \geq t_{0} \geq 0,
$$

where $A(\cdot), B(\cdot) \in \mathbb{R}^{n \times n}$ are matrices whose elements are continuous functions on $\mathbb{R}_{+}$and $F: \mathbb{R}_{+} \times \mathbb{R}^{n} \rightarrow \mathbb{R}^{n}$ is continuous in time and locally Lipschitz in $x$, uniformly in $t$. The system (3.1) is seen as a perturbation of the nominal system:

$$
\dot{x}=A(t) x .
$$

The goal is to represent the perturbation as an additive term on the right-hand side of the state equation. The perturbation terms $B(t)$ and $F(t, x)$ could result from errors in modelling the nonlinear system, ageing of parameters, or uncertainties and disturbances which exist in any realistic problem. We will direct our special attention to the perturbed term $F(t, x)$. In a typical situation, we do not know $F(t, x)$, but we know some information about it, like knowing an upper bound on $\|F(t, x)\|$. The trivial question posed here is: If the linear nominal system presents one of the stability types, the perturbed one keeps the same behavior or not? In this study, we give a condition on $B(t)$ and we introduce two cases on $F(t, x)$. The first one is when $F(t, 0)=0$, while the second case is when $F(t, 0) \neq 0$.

We start this section by showing the global existence and uniqueness of solutions to system (3.1). Further, we give sufficient conditions on the perturbed terms in which $F(t, 0)=0$ to investigate the global uniform $h$-stability of system (3.1).

Lemma 3.1. Consider the differential system (3.1) and suppose that there exist continuous functions $\alpha$ and $\eta$ on $\mathbb{R}_{+}$, such that

$$
\|F(t, x)\| \leq \eta(t) \alpha(\|x\|), \quad \forall x \in \mathbb{R}^{n}, \quad \forall t \geq 0,
$$

and there exists a positive constant $M$ satisfying

$$
\alpha(\|x\|) \leq M\|x\|, \quad \forall x \in \mathbb{R}^{n} .
$$

Then, for all $\left(t_{0}, x_{0}\right) \in \mathbb{R}_{+} \times \mathbb{R}^{n}$, there exists a unique maximal solution $x(\cdot)$ to system (3.1), such that $x\left(t_{0}\right)=x_{0}$. Moreover, the solution $x(\cdot)$ is defined on $\left[t_{0},+\infty\right)$.

Proof. The system (3.1) can be written as $\dot{x}=f(t, x)$, where

$$
f(t, x)=A(t) x+B(t) x+F(t, x) .
$$

The function $f$ is continuous in $t$ and locally Lipschitz in $x$; thus, for all $\left(t_{0}, x_{0}\right) \in$ $\mathbb{R}_{+} \times \mathbb{R}^{n}$, there exists a unique solution $x(\cdot)$ of system $(3.1)$, such that $x\left(t_{0}\right)=x_{0}$. We prove next that $x(\cdot)$ is defined on $\left[t_{0},+\infty\right)$. Assume that is not true, that is, there exists $T_{\max } \in\left(t_{0},+\infty\right)$, such that $x(\cdot)$ is defined on $\left[t_{0}, T_{\max }\right)$. Then, for all $t \in\left[t_{0}, T_{\max }\right)$, we have

$$
\|\dot{x}(t)\| \leq\left(k_{1}+k_{2} M\right)\|x(t)\|
$$

where

$$
k_{1}=\sup _{\left[t_{0}, T_{\max }\right]}\|A(t)+B(t)\|, \quad k_{2}=\sup _{\left[t_{0}, T_{\max }\right]}\|\eta(t)\|
$$


Therefore,

$$
\left\|\int_{t_{0}}^{t} \dot{x}(s) d s\right\| \leq \int_{t_{0}}^{t}\left(k_{1}+k_{2} M\right)\|x(s)\| d s .
$$

Thus, for all $t \in\left[t_{0}, T_{\max }\right)$, we get

$$
\begin{aligned}
\|x(t)\| & \leq e^{\int_{t_{0}}^{t}\left(k_{1}+k_{2} M\right) d s} \\
& \leq k_{3},
\end{aligned}
$$

with

$$
k_{3}=e^{\left(k_{1}+k_{2} M\right) T_{\max }} .
$$

This yields that $x(\cdot)$ remains within the compact $\mathcal{B}_{k_{3}}$, which contradicts our assumption, that is,

$$
\text { if } \quad T_{\max }<+\infty \Longrightarrow \lim _{t \rightarrow+\infty}\|x(t)\|=+\infty .
$$

Therefore, $T_{\max }=+\infty$ and the lemma is proved.

Now, we are ready to present an auxiliary but fundamental result.

Theorem 3.2. Assume that the linear system (3.2) is globally uniformly $h$ stable. Suppose that there exists $\gamma$, a non-negative continuous integrable function on $\mathbb{R}_{+}$, such that

$$
\|B(t)\|_{i} \leq \gamma(t), \quad \forall t \geq 0
$$

and a function $\eta$ which is non-negative continuous and integrable on $\mathbb{R}_{+}$satisfies

$$
\|F(t, x)\| \leq \eta(t) \alpha(\|x\|), \quad \forall x \in \mathbb{R}^{n}, \quad \forall t \geq 0,
$$

where $\alpha$ is a non-negative continuous function on $\mathbb{R}_{+}$verifying

$$
\alpha(\|x\|) \leq M\|x\|, \quad M>0, \quad \forall x \in \mathbb{R}^{n} .
$$

Then, the perturbed system (3.1) is globally uniformly $\tilde{h}$-stable, where $\tilde{h}(t)=$ $h(t) e^{c \int_{t_{0}}^{t}(\gamma(s)+M \eta(s)) d s}$ with $c \geq 1$.

Proof. Let $x(t)$ be the solution to system (3.1), then

$$
x(t)=R\left(t, t_{0}\right) x_{0}+\int_{t_{0}}^{t} R(t, s)(B(s) x(s)+F(s, x(s))) d s,
$$

where $R\left(t, t_{0}\right)$ is the transition matrix of the linear system (3.2). Hence,

$$
h(t)^{-1}\|x(t)\| \leq \operatorname{ch}\left(t_{0}\right)^{-1}\left\|x_{0}\right\|+c \int_{t_{0}}^{t}(\gamma(s)+M \eta(s)) h(s)^{-1}\|x(s)\| d s .
$$

Putting $u(t)=h(t)^{-1}\|x(t)\|$, we get

$$
u(t) \leq c u\left(t_{0}\right)+c \int_{t_{0}}^{t}(\gamma(s)+M \eta(s)) u(s) d s .
$$

According to Lemma 2.17, we obtain for all $t \geq t_{0}$

$$
u(t) \leq c u\left(t_{0}\right) e^{c \int_{t_{0}}^{t}(\gamma(s)+M \eta(s)) d s} .
$$


Therefore, for all $t \geq t_{0}$ and all $x_{0} \in \mathbb{R}^{n}$, the solution to the system (3.1) is given by:

$$
\|x(t)\| \leq c\left\|x_{0}\right\| \tilde{h}(t) \tilde{h}\left(t_{0}\right)^{-1}
$$

This finishes the proof.

As a particular case of the previous theorem, we have the following result.

Corollary 3.3. We consider the differential system:

$$
\dot{x}=A(t) x+B(t) x, \quad x\left(t_{0}\right)=x_{0}, \quad t \geq t_{0} \geq 0,
$$

with the system (3.2) is globally uniformly h-stable and $B(t)$ satisfies:

$$
\int_{0}^{+\infty}\|B(s)\| d s<\infty .
$$

Then, the system (4.2) is also globally uniformly h-stable.

Remark 3.4. Similar conclusion can be drawn when $B(t) \rightarrow 0$ as $t \rightarrow+\infty$.

As an application of the previous corollary, we study the following perturbed linear harmonic oscillator. It is a common model used in physics because of the wide range of problems it can be applied to. The linear harmonic oscillator describes vibrations in molecules and their counterparts in solids, the phonons. Many more physical systems can, at least approximately, be described in terms of linear harmonic oscillator models. However, the most eminent role of this oscillator is its linkage to the boson, one of the conceptual building blocks of microscopic physics.

Example 3.5. Consider a damped harmonic oscillator, as shown in Figure 2. The dynamics of the system are given by the equation:

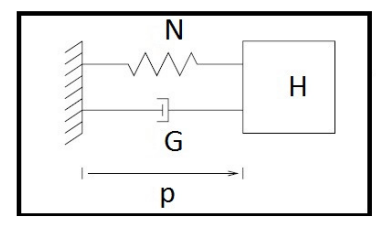

Figure 2. Damped harmonic oscillator.

$$
H \ddot{p}+G \dot{p}+\frac{L}{(1+t)^{2}} p+N p=0,
$$

where $t \in \mathbb{R}_{+}$is the time, $H, G$ and $N$ are all positive quantities. As a state space equation, we rewrite the equation (3.6) as

$$
\left\{\begin{array}{l}
\dot{p}=\dot{p} \\
\ddot{p}=-\frac{N}{H} p-\frac{G}{H} \dot{p}-\frac{L}{H(1+t)^{2}} p .
\end{array}\right.
$$

We define $X=(p, \dot{p}) \in \mathbb{R}^{2}$ as the state of the system. The system (3.7) can be written as follows:

$$
\dot{X}=A(t) X+B(t) X, \quad \forall t \geq 0
$$


where

$$
A(t)=A=\left(\begin{array}{cc}
0 & 1 \\
-\frac{N}{H} & -\frac{G}{H}
\end{array}\right), \quad B(t)=\left(\begin{array}{cc}
0 & 0 \\
-\frac{L}{H(1+t)^{2}} & 0
\end{array}\right) .
$$

The characteristic equation of the linear system $\dot{X}=A(t) X$ is given by:

$$
\lambda^{2}+\frac{G}{H} \lambda+\frac{N}{H}=0 .
$$

Then,

$$
\lambda_{1,2}=\frac{-G \pm \sqrt{G^{2}-4 N H}}{2 H},
$$

which always have negative real parts, hence the system is globally uniformly $h$-stable. Moreover,

$$
\|B(t)\|_{i}=\frac{L}{H} \frac{1}{(1+t)^{2}}, \quad \forall t \geq 0 .
$$

Put $\gamma(t)=\frac{L}{H} \frac{1}{(1+t)^{2}}$, which is non-negative continuous and integrable on $\mathbb{R}_{+}$. Therefore, from Corollary 3.3, one can conclude that the system (3.6) is globally uniformly $h$-stable.

\section{UNIFORM BOUNDEDNESS OF PERTURBED SYSTEMS}

The origin may not be an equilibrium point of the perturbed system (3.1). We can no longer study $h$-stability of the origin as an equilibrium point, nor should we expect the solution to the perturbed system to approach the origin as $t \rightarrow \infty$, the best we can hope is the bounded and the asymptotic behavior of solutions. For that, if the perturbed term $F(t, x)$ is small in some sense, then $x(t)$ will be uniformly bounded by a small bound, that is, $\|x(t)\|$ will be small for sufficiently large $t$.

In what follows, we will show the global existence and uniqueness of solutions to system (3.1). In addition, we will investigate the global uniform boundedness of the perturbed system under the conditions on the right hand side of the system via the Gronwall-Bellman Type Integral Inequality.

Lemma 4.1. Consider the nonlinear system (3.1) and assume that there exist continuous functions $h$ and $\rho$ on $\mathbb{R}_{+}$, such that

$$
\|F(t, x)\| \leq h(t) \rho(t), \quad \forall t \geq 0 .
$$

Then, for all $\left(t_{0}, x_{0}\right) \in \mathbb{R}_{+} \times \mathbb{R}^{n}$, there exists a unique maximal solution $x(\cdot)$ to system (3.1), such that $x\left(t_{0}\right)=x_{0}$. Further, the solution $x(\cdot)$ is defined on $\left[t_{0},+\infty\right)$.

Proof. We can write the system (3.1) as $\dot{x}=f(t, x)$, with

$$
f(t, x)=A(t) x+B(t) x+F(t, x) .
$$

The function $f$ is continuous in $t$ and locally Lipschitz in $x$, then, for all $\left(t_{0}, x_{0}\right) \in$ $\mathbb{R}_{+} \times \mathbb{R}^{n}$, there exists a unique solution $x(\cdot)$ to system $(3.1)$, such that $x\left(t_{0}\right)=x_{0}$. We prove now that $x(\cdot)$ is defined on $\left[t_{0},+\infty\right)$. Suppose that is not true, that is, 
there exists $T_{\max } \in\left(t_{0},+\infty\right)$, such that $x(\cdot)$ is defined on $\left[t_{0}, T_{\max }\right)$. So, for all $t \in\left[t_{0}, T_{\max }\right)$, we have

$$
\|\dot{x}(t)\| \leq k_{1}\|x(t)\|+k_{2}
$$

where

$$
k_{1}=\sup _{\left[t_{0}, T_{\max }\right]}\|A(t)+B(t)\|, \quad k_{2}=\sup _{\left[t_{0}, T_{\max }\right]}\|h(t) \rho(t)\| .
$$

Therefore,

$$
\left\|\int_{t_{0}}^{t} \dot{x}(s) d s\right\| \leq \int_{t_{0}}^{t}\left[k_{1}\|x(s)\|+k_{2}\right] d s .
$$

Thus, for all $t \in\left[t_{0}, T_{\max }\right)$, we get

$$
\begin{aligned}
\|x(t)\| & \leq k_{2} e^{\int_{t_{0}}^{t} k_{1} d s} \\
& \leq k_{3},
\end{aligned}
$$

with

$$
k_{3}=k_{2} e^{k_{1} T_{\max }} .
$$

This yields that $x(\cdot)$ remains within the compact $\mathcal{B}_{k_{3}}$, which contradicts our assumption, that is,

$$
\text { if } \quad T_{\max }<+\infty \Longrightarrow \lim _{t \rightarrow \infty}\|x(t)\|=+\infty .
$$

Consequently, $T_{\max }=+\infty$. The proof is completed.

One has the following theorem.

Theorem 4.2. Suppose that the linear time-varying system (3.2) is globally uniformly $h$-stable with $h$ being a decreasing function on $\mathbb{R}_{+}$. Assume that there exists $\gamma$, a non-negative continuous integrable function on $\mathbb{R}_{+}$, such that

$$
\|B(t)\|_{i} \leq \gamma(t), \quad \forall t \geq 0
$$

and a non-negative continuous and integrable function $\rho(t) \in \mathbb{R}_{+}$satisfies

$$
\|F(t, x)\| \leq h(t) \rho(t), \quad \forall x \in \mathbb{R}^{n}, \quad \forall t \geq 0 .
$$

Then, the solutions to system (3.1) are globally uniformly bounded.

Proof. Let $x(t)$ be the solution to system (3.1), then we have

$$
x(t)=R\left(t, t_{0}\right) x_{0}+\int_{t_{0}}^{t} R(t, s)[B(s) x(s)+F(s, x(s))] d s,
$$

where $R\left(t, t_{0}\right)$ is the transition matrix of the linear system (3.2). Thus,

$$
h(t)^{-1}\|x(t)\| \leq c\left\|x_{0}\right\| h\left(t_{0}\right)^{-1}+c \int_{t_{0}}^{t} h(s)^{-1}[\gamma(s)\|x(s)\|+h(s) \rho(s)] d s .
$$

Put $u(t)=h(t)^{-1}\|x(t)\|$, then

$$
u(t) \leq c u\left(t_{0}\right)+c \int_{t_{0}}^{t}[\gamma(s) u(s)+\rho(s)] d s .
$$

Applying Lemma 2.16, one can get for all $t \geq t_{0}$

$$
\begin{aligned}
u(t) & \leq c u\left(t_{0}\right) e^{c \int_{t_{0}}^{t} \gamma(s) d s}+\kappa e^{c \int_{t_{0}}^{t}\left(\gamma(s)+\frac{1}{\kappa} \rho(s)\right) d s}, \quad \forall \kappa>0 \\
& \leq c M_{1} u\left(t_{0}\right)+\kappa M_{1} M_{2},
\end{aligned}
$$


such that $M_{1}=e^{c \int_{0}^{\infty} \gamma(s) d s}$ and $M_{2}=e^{\frac{c}{\kappa} \int_{0}^{\infty} \rho(s) d s}$. Then,

$$
\|x(t)\| \leq c M_{1}\left\|x_{0}\right\| h(t) h\left(t_{0}\right)^{-1}+\kappa M_{1} M_{2}\|h\|_{\infty},
$$

where $\|h\|_{\infty}=\sup _{t \geq 0}\{h(t)\}$. Consequently, the solutions to system (3.1) are globally uniformly bounded.

Note that the last inequality implies that

$$
\|x(t)\|-\kappa M_{1} M_{2}\|h\|_{\infty} \leq c M_{1}\left\|x_{0}\right\| h(t) h\left(t_{0}\right)^{-1} .
$$

So, for all $t \geq 0$ and for all $\kappa>0$ small enough, if we take $\left\|x_{0}\right\| \geq \kappa M_{1} M_{2}\|h\|_{\infty}$, such that $\|x(t)\| \geq \kappa M_{1} M_{2}\|h\|_{\infty}$, we have the solutions to system (3.1) approaching, when $t$ goes to infinity, the compact set $S$ defined by

$$
S=\left\{x \in \mathbb{R}^{n},\|x\| \leq \kappa M_{1} M_{2}\|h\|_{\infty}\right\} .
$$

This ends the proof.

\section{EXAMPLES}

In this section, we introduce some examples with simulations to illustrate the effectiveness of the obtained results.

Example 5.1. We consider the two-dimensional differential equation:

$$
\left\{\begin{array}{l}
\dot{x}_{1}=-\frac{t}{2} x_{1}+x_{2}+t e^{-t} x_{1}+\frac{x_{1}}{1+t^{2}}, \\
\dot{x}_{2}=-x_{1}-\frac{t}{2} x_{2}
\end{array}\right.
$$

with $x=\left(x_{1}, x_{2}\right)^{T} \in \mathbb{R}^{2}$ and $t \in \mathbb{R}_{+}$. The system (5.1) has the same form as (3.1), where

$$
A(t)=\left(\begin{array}{rc}
-\frac{t}{2} & 1 \\
-1 & -\frac{t}{2}
\end{array}\right), \quad B(t)=\left(\begin{array}{cc}
t e^{-t} & 0 \\
0 & 0
\end{array}\right),
$$

and

$$
F(t, x)=\left(\begin{array}{c}
\frac{x_{1}}{1+t^{2}} \\
0
\end{array}\right) .
$$

The state transition matrix $R\left(t, t_{0}\right)$ of the linear system $\dot{x}=A(t) x$ is given by

$$
R\left(t, t_{0}\right)=e^{-\frac{1}{4}\left(t^{2}-t_{0}^{2}\right)} \omega\left(t-t_{0}\right)
$$

with

$$
\omega(t)=\left(\begin{array}{cc}
\cos t & \sin t \\
-\sin t & \cos t
\end{array}\right)
$$

Then,

$$
\left\|R\left(t, t_{0}\right)\right\|_{i} \leq e^{-\frac{1}{4}\left(t^{2}-t_{0}^{2}\right)}, \quad \forall t \geq t_{0} .
$$

Thus, the linear nominal system is globally uniformly $h$-stable with $h(t)=e^{-\frac{1}{4} t^{2}}$. On one side, the perturbation $F(t, x)$ satisfies the condition (3.3) with $\eta(t)=$ 
$\frac{1}{1+t^{2}}$ which is a non-negative continuous integrable function on $\mathbb{R}_{+}$and $\alpha(\|x\|)=$ $\|x\|$, which verifies (3.4). On the other side, we have

$$
\|B(t)\|_{i} \leq t e^{-t}=\gamma(t), \quad \forall t \geq 0,
$$

such that $\gamma$ is non-negative continuous and integrable on $\mathbb{R}_{+}$. Consequently, from Theorem 3.2, we deduce that the system (5.1) is globally uniformly $\tilde{h}$-stable, where $\tilde{h}(t)=e^{-\frac{1}{4} t^{2}} \exp \left(1-(t+1) e^{-t}+\arctan (t)\right)$.

The trajectory of system (5.1), with respect to the initial state $\left(x_{1}(0), x_{2}(0)\right)=$ $(2,2)$, is depicted in Figure 3 .
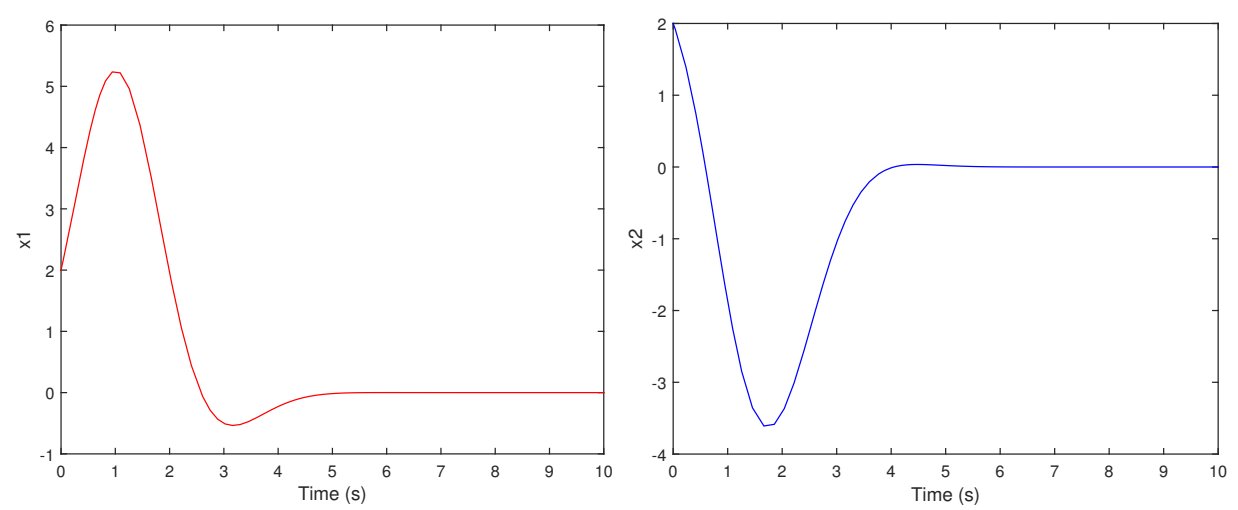

Figure 3. Time evolution of the state $x(t)=\left(x_{1}(t), x_{2}(t)\right)$ of system (5.1).

Example 5.2. We consider the first order problem:

$$
\dot{x}=-\frac{x}{2(1+t)}+\frac{x}{(2+t)(\ln (2+t))^{2}}+\frac{2 x e^{-t}}{1+x^{2}} \sin (t), \quad x \in \mathbb{R}, \quad t \geq 0,
$$

which has the same form as system (3.1), where

$$
A(t)=-\frac{1}{2(1+t)}, \quad B(t)=\frac{1}{(2+t)(\ln (2+t))^{2}}
$$

and

$$
F(t, x)=\frac{2 x e^{-t}}{1+x^{2}} \sin (t) .
$$

Then, the solution to the linear system $\dot{x}=A(t) x$ is given by

$$
x(t)=x_{0}\left(\frac{1+t_{0}}{1+t}\right)^{\frac{1}{2}} .
$$

Therefore, the linear nominal system is globally uniformly $h$-stable with

$$
h(t)=\frac{1}{\sqrt{1+t}} .
$$


Further, the perturbation $F(t, x)$ satisfies the condition (3.3) with $\eta(t)=e^{-t}$, which is a non-negative continuous and integrable function on $\mathbb{R}_{+}$and the function $\alpha$ verifies (3.4), where $\alpha(|x|)=2 \sin (t)|x|$. Moreover,

$$
|B(t)|_{i}=\frac{1}{(2+t)(\ln (2+t))^{2}}, \quad \forall t \geq 0 .
$$

Let

$$
\gamma(t)=\frac{1}{(2+t)(\ln (2+t))^{2}}
$$

which is a non-negative continuous and integrable function on $\mathbb{R}_{+}$. Consequently, we deduce that all hypotheses of Theorem 3.2 are fulfilled, then the system (5.2) is globally uniformly $\tilde{h}$-stable, where

$$
\tilde{h}(t)=\frac{1}{\sqrt{1+t}} \exp \left(2-2 e^{-t}-\frac{1}{\ln (2+t)}+\frac{1}{\ln 2}\right) .
$$

The trajectory of system $(5.2)$, with respect to the initial state $x(0)=1$, is depicted in Figure 4.

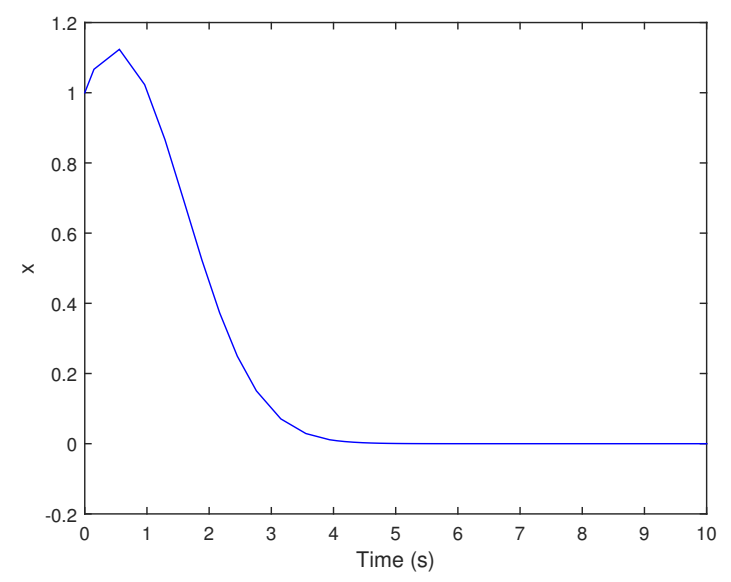

Figure 4. Time evolution of the state $x(t)$ of system (5.2).

Example 5.3. We consider the following scalar equation:

$$
\dot{x}=-\frac{x}{1+t^{2}}+e^{-2 t} x+\frac{\exp (-\arctan (t))}{(1+t)^{3}\left(1+x^{2}\right)}, \quad x \in \mathbb{R}, \quad t \geq 0,
$$

which has the same form as system (3.1), where

$$
A(t)=-\frac{1}{1+t^{2}}, \quad B(t)=e^{-2 t}, \quad F(t, x)=\frac{\exp (-\arctan (t))}{(1+t)^{3}\left(1+x^{2}\right)} .
$$

The solution to the linear system $\dot{x}=A(t) x$ is given by

$$
x(t)=x\left(t_{0}\right) \exp (-\arctan (t)) \exp \left(\arctan \left(t_{0}\right)\right) .
$$


Therefore, the linear nominal system is globally uniformly $h$-stable with $h(t)=$ $\exp (-\arctan (t))$, which is decreasing on $\mathbb{R}_{+}$. On the other hand, one has

$$
|F(t, x)| \leq \frac{\exp (-\arctan (t))}{(1+t)^{3}}=h(t) \rho(t), \quad \forall t \geq 0,
$$

where

$$
\rho(t)=\frac{1}{(1+t)^{3}},
$$

which is a non-negative continuous and integrable function on $\mathbb{R}_{+}$. In addition, one has

$$
|B(t)|_{i} \leq e^{-2 t}, \quad \forall t \geq 0 .
$$

Let $\gamma(t)=e^{-2 t}$, which is non-negative continuous and integrable on $\mathbb{R}_{+}$. Therefore, all the conditions of Theorem 4.2 are satisfied. Then, the solutions to system (5.3) are globally uniformly bounded.

Moreover, for all $t \in \mathbb{R}_{+}$and for $\kappa>0$ small enough, if we take $|x(0)| \geq$ $\kappa e^{\frac{1}{2}\left(1+\frac{1}{\kappa}\right)-\frac{\pi}{2}}$, such that $|x(t)| \geq \kappa e^{\frac{1}{2}\left(1+\frac{1}{\kappa}\right)-\frac{\pi}{2}}$, then we obtain that the solutions to system (5.3) approach, when $t \rightarrow+\infty$, the compact set $S$ defined by

$$
S=\left\{x \in \mathbb{R},|x| \leq \kappa e^{\frac{1}{2}\left(1+\frac{1}{\kappa}\right)-\frac{\pi}{2}}\right\} .
$$

The trajectory of system (5.3), with respect to the initial state $x(0)=2$, is depicted in Figure 5.

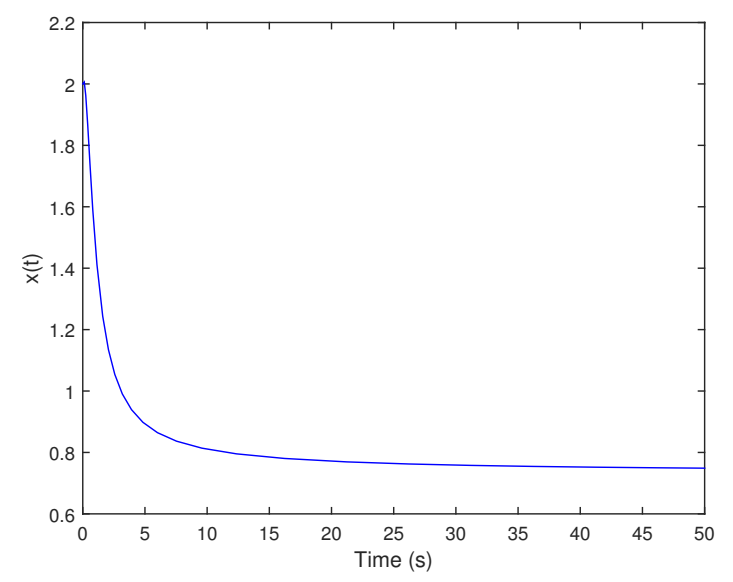

Figure 5. Time evolution of the state $x(t)$ of the system (5.3).

\section{Conclusion}

Sufficient conditions are presented to investigate the global uniform $h$-stability and the global uniform boundedness of such perturbed differential equations with the global existence and uniqueness of solutions based on certain Gronwall integral inequalities. Some examples and simulations are given to illustrate the validity of the obtained results. 


\section{References}

[1] A. BenAbdallah, I. Ellouze and M. A. Hammami, Practical stability of nonlinear timevarying cascade systems, J. Dyn. Control Syst. 15 (2009), 45-62.

[2] A. Ben Makhlouf and M. A. Hammami, A nonlinear inequality and application to global asymptotic stability of perturbed systems, Math. Method. Appl. Sci. 38 (2015), 2496-2505.

[3] P. Bohl, Über Differentialgleichungen, J. für Math. 144, 284-313. (1914) 144 (1913), 284313.

[4] H. Damak, M. A. Hammami and A. Kicha, A converse theorem on practical h-stability of nonlinear systems, Mediterr. J. Math. 17 (2020), Paper No. 88, 18 pp.

[5] H. Damak, M. A. Hammami and A. Kicha, A converse theorem for practical h-stability of time-varying nonlinear systems, N. Z. J. Math. 50 (2020), 109-123.

[6] H. Damak, M. A. Hammami and A. Kicha, Growth conditions for asymptotic behavior of solutions for certain time-varying differential equations, Differential Equations and Control Processes 2021, No. 1 (2021), 423-447.

[7] T.H. Gronwall, Note on the derivatives with respect to a parameter of the solutions to a system of differential equations, Annals of Math. Second Ser. 20 (1919), 292-296.

[8] M. Hammi and M. A. Hammami, Non-linear integral inequalities and applications to asymptotic stability, IMA J. Math. Control Inf. 32 (2015), 717-735.

[9] M. Hammi and M. A. Hammami, Gronwall-Bellman type integral inequalities and applications to global uniform asymptotic stability, Cubo 17 (2015), 53-70.

[10] B. Ben Hamed, Z. Haj Salem and M. A. Hammami, Stability of nonlinear time-varying perturbed differential equations, Nonlinear Dyn. 73 (2013), 1353-1365.

[11] N.A. Izobov, Linear systems of ordinary differential equations, J. Sov. Math. 5 (1974), 46-96.

[12] H. K. Khalil, Nonlinear Systems, Prentice Hall, New York, 2002.

[13] R. Medina and M. Pinto, Stability of nonlinear difference equations, in: G. S. Ladde et al. (eds.), Dynamic Systems and Applications, Vol. 2., Proceedings of the 2nd International Conference, Morehouse College, Atlanta, GA, USA, May 24-27, 1995, Dynamic Publishers, Atlanta, GA, 1996, pp. 397-404.

[14] M. Pinto, Stability of nonlinear differential systems, Appl. Anal. 43 (1992), 1-20.

[15] M. Pinto, Perturbations of asymptotically stable differential systems, Analysis 4 (1984), 161-175.

[16] M. Vidyasagar, Nonlinear Systems Analysis, Classics in Applied Mathematics, Society for Industrial and Applied Mathematics, Philadelphia, PA, 2002.

[17] R. È. Vinograd, Estimation of the jump of the higher characteristic exponent in the case of small perturbations, Doklady Akademii Nauk SSSR 114 (1957), 459-461.

[18] R. È. Vinograd, Simultaneous attainability of central Lyapunov and Bohl exponents for ODE linear systems, Proceedings of the American Mathematical Society 88 (1983), 595-601.

Hanen Damak, Department of Mathematics, University of Sfax, Faculty of Sciences of Sfax, Route Soukra BP1171, 3000 Sfax, Tunisia

e-mail: hanen.damak@yahoo.fr

Mohamed Ali Hammami, Department of Mathematics, University of Sfax, Faculty of Sciences of Sfax, Route Soukra BP1171, 3000 Sfax, Tunisia

e-mail: mohamedali.hammami@fss.rnu.tn

Abir Kicha, Department of Mathematics, University of Sfax, Faculty of Sciences of Sfax, Route Soukra BP1171, 3000 Sfax, Tunisia

e-mail: kichaabir05@gmail.com 
